\title{
Processo de indicação do implante coclear em uma criança com paralisia cerebral: estudo de caso
}

\author{
Indication process of cochlear implant for a child with cerebral \\ palsy: a case report
}

\author{
Maria Jaquelini Dias dos Santos¹, Maria Cecília Bevilacqua², Adriane Lima Mortari Moret², \\ Dionísia Aparecida Cusin Lamônica ${ }^{2}$, Orozimbo Alves Costa ${ }^{2}$, Elisabete Honda Yamaguti ${ }^{3}$
}

\begin{abstract}
RESUMO
A paralisia cerebral (PC) é o transtorno motor mais frequente na infância, podendo apresentar outras deficiências associadas, inclusive a deficiência auditiva. Estudos sobre a indicação do implante coclear (IC) em crianças com PC e deficiência auditiva são restritos internacionalmente, e no Brasil, inexistentes. Desta maneira, o objetivo do estudo foi descrever o processo de avaliação pré-cirúrgica de uma criança com PC candidata ao IC. O estudo foi realizado com um menino, com 2 anos e 6 meses de idade, com PC e deficiência auditiva bilateral. O protocolo pré-cirúrgico seguiu os procedimentos já utilizados no Hospital de Reabilitação de Anomalias Craniofaciais, compreendendo: avaliações fonoaudiológicas, neurológica, otorrinolaringológica e psicológica, por meio de observações clinicas, questionários, e exames radiológicos. O paciente foi diagnosticado com espectro da neuropatia auditiva de grau profundo bilateral, quadriparesia atetóide de grau moderado, atraso global do desenvolvimento, exceto na função visual, comportamento comunicativo intencional presente, bom contato social, ausência de comprometimentos intelectuais ou outras deficiências associadas ao quadro da PC e família integrada ao tratamento. De acordo com os resultados obtidos, o paciente foi considerado apto para cirurgia de IC, e permanece em acompanhamento sistemático no respectivo programa. Ressalta-se a importância de uma etapa pré-cirúrgica ampla e estruturada para crianças com paralisia cerebral e candidatas ao IC. Por meio de instrumentos de avaliação direcionados aos aspectos globais do desenvolvimento, é possível obter informações específicas que enriquecem as orientações dadas aos pais sobre o prognóstico da criança e possibilitam traçar metas reais de reabilitação auditiva.
\end{abstract}

Descritores: Implante coclear; Paralisia cerebral; Perda auditiva; Criança; Avaliação, Deficiências do desenvolvimento

\section{INTRODUÇÃO}

A paralisia cerebral (PC) é definida como sequela de lesão encefálica de base etiológica não evolutiva, com sinais e sintomas variáveis. Caracteriza-se por ser o transtorno motor mais frequente na infância ${ }^{(1-3)}$, podendo estar presentes alterações associadas em diferentes funções: visual, mental, emocional ou auditiva $^{(1)}$. Pelo menos $12 \%$ das crianças com PC, apresentam

Trabalho realizado no Centro de Pesquisas Audiológicas do Hospital de Reabilitação de Anomalias Craniofaciais, Universidade de São Paulo - USP Bauru (SP), Brasil.

(1) Programa de Pós-graduação (Mestrado) em Fonoaudiologia, Departamento de Fonoaudiologia, Faculdade de Odontologia de Bauru, Universidade de São Paulo - USP - Bauru (SP), Brasil.

(2) Departamento de Fonoaudiologia, Faculdade de Odontologia de Bauru, Universidade de São Paulo - USP - Bauru (SP), Brasil.

(3) Centro de Pesquisas Audiológicas, Hospital de Reabilitação de Anomalias Craniofaciais, Universidade de São Paulo - USP - Bauru (SP), Brasil.

Endereço para correspondência: Maria Jaquelini Dias dos Santos. R. Sílvio

Marchione, 320, Vila Universitária, Bauru (SP), Brasil, CEP: 17012-900.

E-mail: mariajaquelini@usp.com

Recebido em: 1/10/2010; Aceito em: 31/3/2011 perda auditiva de caráter sensorioneural ${ }^{(2)}$.

Internacionalmente, o implante coclear (IC) tem se destacado pelo potencial de habilitação e reabilitação das habilidades auditivas, bem como da linguagem oral ${ }^{(4-6)}$. Para crianças que apresentam a deficiência auditiva isolada, vários estudos na literatura nacional e internacional relatam os ótimos resultados. No entanto, para crianças com deficiências associadas à surdez, em especial a PC, a indicação do IC, ainda é pouco estudada $^{(2,3,5,7,8)}$.

Vários aspectos são ponderados quando, além da deficiência auditiva, outras condições estão presentes na criança. Cada desabilidade adicional à surdez caracterizará um quadro clínico distinto, que influenciará desde a conduta diagnóstica até a (re)habilitação da deficiência auditiva. Os resultados auditivos dependerão, dentre outros fatores, do potencial da criança, no que se refere ao seu desenvolvimento global.

Estudos internacionais sobre o IC em crianças com PC são restritos ${ }^{(2,3,5,7,8)}$, e em nível nacional, estes são inexistentes. Deste modo, o objetivo do estudo é descrever o processo de avaliação pré-cirúrgica em uma criança com PC, candidata ao IC. 


\section{APRESENTAÇÃO DO CASO CLÍNICO}

Esta pesquisa foi aprovada pelo Comitê de Ética em Pesquisa do Hospital de Reabilitação de Anomalias Craniofaciais da Universidade de São Paulo (USP), processo ${ }^{\circ}$ 041/2008, e recebeu apoio do Conselho Nacional de Desenvolvimento Científico e Tecnológico (CNPq), processo no 116125/2008 0.

A pesquisa foi realizada no Centro de Pesquisas Audiológicas do Hospital de Reabilitação de Anomalias Craniofaciais da USP. Para início do processo de avaliação, o representante legal da criança, assinou o Termo de Consentimento Livre Esclarecido, seguindo a resolução MS/CNS/CNEP nº 196/96 de 10 de outubro de 1996.

\section{Caracterização do paciente da pesquisa}

Menino, com 2 anos e 6 meses de idade na data das avaliações pré-cirúrgicas. Nasceu pré-termo de 34 semanas, com histórico de anóxia, icterícia, uso de ototóxicos, e permanência na Unidade de Tratamento Intensivo (UTI) por 15 dias.

Aproximadamente aos 10 meses de idade o paciente foi diagnosticado com deficiência auditiva bilateral de grau profundo, sendo já possível observar atraso no desenvolvimento neuropsicomotor, com suspeita de paralisia cerebral (PC). A intervenção audiológica ocorreu aos 18 meses de idade, com o aparelho de amplificação sonora individual (AASI), e acompanhamento sistemático em programa de reabilitação fonoaudiológica, com enfoque na comunicação oral, em sua cidade de origem.

O paciente chegou ao Programa de Implante Coclear do hospital, aos 2 anos de idade, já adaptado aos aparelhos de amplificação sonora individuais e inserido em um programa de reabilitação motora desde os 12 meses de idade. Já estava também matriculado como aluno de escola regular infantil.

\section{Protocolo de avaliação pré-cirúrgica para crianças com paralisia cerebral}

Os critérios institucionais utilizados durante a fase pré-cirúrgica foram considerados para o estudo deste paciente ${ }^{(4)}$. O processo de avaliação pré-cirúrgica foi realizado por uma equipe interdisciplinar, formada por fonoaudiólogos, médicos, psicólogos, e assistentes sociais. A avaliação fonoaudiológica compreendeu os seguintes procedimentos: Audiometria de Reforço Visual (ARV), Limiar de Detecção Verbal (LDV), Imitanciometria (I), Emissões Otoacústicas Evocadas transiente e produto de distorção (EOEt/dp), Potenciais Evocados Auditivos de Troco Encefálico (PEATE), Resposta Auditiva de Estado Estável (RAEE); e aplicação dos seguintes questionários e escalas: Infant Toddler Meaningful Auditory Infant Scale (IT-MAIS) ${ }^{(10)}$, Meaningful Useful Speech Scale (MUSS) ${ }^{(6)}$, Production Infant Scale Evaluation (PRISE) ${ }^{(11)}$, Inventário MacArthur: primeiras palavras e gestos ${ }^{(12)}$, Escala de Gesel e Amatruda ${ }^{(13)}$, Early Language Milestone Scale (ELM) ${ }^{(14)}$. Após a aplicação destes procedimentos, foram atribuídas as Categorias de Audição ${ }^{(4)}$, de Linguagem Expressiva ${ }^{(4)}$, e classificação quanto ao Modo de Comunicação predominante ${ }^{(3)}$.
As avaliações médicas compreenderam as áreas da otorrinolaringologia e da neurologia. Foram realizados exames clínicos e de imagem: tomografia computadorizada das mastóides (TC), ressonância magnética de crânio, da fossa posterior e regiões temporais (RM).

A área de psicologia avaliou o paciente e sua família. Aliada à entrevista psicológica estruturada, utilizou-se a classificação quanto ao grau de permeabilidade da família e ao estilo cognitivo da criança $a^{(4)}$.

\section{RESULTADOS}

A análise dos achados audiológicos na ARV, EOEt/pd, PEATE, RAEE, e imitanciometria (Tabela 1), indicaram perda auditiva sensorioneural de grau profundo bilateral, caracterizando-a como desordem do espectro da neuropatia auditiva. Aliados aos resultados audiológicos, os achados na RM de fossa posterior e regiões temporais, descartaram hipoplasiaou agenesia do nervo auditivo. Tais condições se presentes, são contra-indicações ao IC.

A perda auditiva apresentada pelo participante do estudo, não é considerada contra-indicação ao IC. A presença de perda auditiva sensorioneural profunda ou severa bilateral é uma das condições para que o indivíduo seja candidato ao IC.

$\mathrm{Na} A R V$, realizada com estímulo warble em campo livre, não foi observada diferença significativa entre os limiares sem e com AASI (Tabela 1). O ganho de $25 \mathrm{~dB}$ para OD e de $20 \mathrm{~dB}$ para OE com o uso do AASI, não foi suficiente para alcançar os limiares de audibilidade necessários para a percepção dos sons de fala. $\mathrm{O}$ mesmo foi observado quanto à detecção de fala (LDV), na qual o ganho foi equivalente para ambas as orelhas: $30 \mathrm{~dB}$ (Tabela 1).

Deve ser considerado, além do ganho em decibels, o comportamento comunicativo do indivíduo perante o mundo sonoro com o AASI. Neste estudo, o participante não apresentou mudança significativa quanto ao comportamento comunicativo durante o uso do AASI, para que este fosse mantido como opção de tratamento para a deficiência auditiva em questão. Contudo, é importante ressaltar que o participante fez uso efetivo do AASI desde os 10 meses de idade, portanto, teve a oportunidade de receber estimulação auditiva por aproximadamente 20 meses antes de receber o IC.

A estimulação auditiva precoce por meio do AASI, que antecede a indicação do IC, favorece o processo de maturação das estruturas do sistema auditivo, pois envia informações sonoras para um sistema neural em formação. Estas informações são armazenadas e passam a constituir o repertório mnemônico do paciente frente ao mundo sonoro, o que não deixa de ser um importante fator para o desenvolvimento das habilidades auditivas, de fala e de linguagem após a cirurgia de IC e, consequentemente, característica importante para a indicação do IC.

Os critérios considerados como contra-indicação ao $\mathrm{IC}^{(9)}$, não foram observados neste paciente; são eles: condições médicas que contra-indicassem a cirurgia; deficiência auditiva causada por agenesia de cóclea, de nervo auditivo ou por lesões centrais; infecção ativa do ouvido médio, comprometimentos graves neurológicos associados à deficiência auditiva. 
Neste estudo, apesar da PC ser considerada como uma entidade neurológica, a mesma não foi considerada impeditiva para o uso de IC, pois a área afetada, neste caso específico, refere-se à área motora.

A avaliação neurológica classificou a PC como quadriparesia atetóide de grau moderado, com maior prejuízo motor no lado esquerdo e membros superiores. A avaliação neurológica e a avaliação psicológica descartaram alterações cognitivas ou condições psicopatológicas em associação à PC que pudessem interferir no processo de habilitação com o IC. Desta forma, acredita-se que o uso do IC pode otimizar as habilidades re- ceptivas e promover integração dos estímulos auditivos, possibilitando o desenvolvimento de habilidades comunicativas.

Sendo assim, os resultados da avaliação pré-cirúrgica foram analisados pela equipe, e considerados favoráveis para a indicação da cirurgia de IC (Tabelas 1 e 2).

A indicação do IC foi discutida com a família do paciente e, após a concordância da família com a realização da cirurgia, o paciente recebeu o IC da marca Advanced Bionics modelo HiRes $90 \mathrm{~K}$, com inserção total dos eletrodos na cóclea esquerda. A idade do paciente na cirurgia era de 2 anos e 7 meses; a ativação do implante coclear foi realizada um mês após a

Tabela 1. Resultados da avaliação pré-cirúrgica utilizado para indicação do implante coclear em crianças com paralisia cerebral: aspectos fonoaudiológicos

\begin{tabular}{|c|c|c|}
\hline Avaliações & Resultados & \\
\hline Avaliação fonoaudiológica & OD & OE \\
\hline \multicolumn{3}{|l|}{ ARV (campo livre) } \\
\hline Sem AASI & $95 \mathrm{~dB}$ & $95 \mathrm{~dB}$ \\
\hline Com AASI & $70 \mathrm{~dB}$ & $75 \mathrm{~dB}$ \\
\hline \multicolumn{3}{|l|}{ LDV (campo livre) } \\
\hline Sem AASI & $85 \mathrm{~dB}$ & $90 \mathrm{~dB}$ \\
\hline Com AASI & $55 \mathrm{~dB}$ & $60 \mathrm{~dB}$ \\
\hline Timpanometria & Curva A & Curva A \\
\hline Pesquisa do reflexo estapediano & Reflexos ausentes & Reflexos ausentes \\
\hline \multicolumn{3}{|l|}{ EOE } \\
\hline Transiente & Ausente & Ausente \\
\hline Produto de distorção & Presente & Presente \\
\hline PEATE & Ausente com microfonismo coclear & Ausente com microfonismo coclear \\
\hline RAEE & Presente em $110 \mathrm{~dB}$ & Presente em $110 \mathrm{~dB}$ \\
\hline \multicolumn{3}{|l|}{ Questionários } \\
\hline IT-MAIS & $40 \%$ & \\
\hline MUSS & $27,5 \%$ & \\
\hline PRISE & $59 \%$ & \\
\hline Inventário MacArthur & Área de predomínio: ações e gestos & \\
\hline \multicolumn{3}{|l|}{ Escalas / Categorias } \\
\hline \multicolumn{3}{|l|}{ Escala ELM } \\
\hline Função auditiva receptiva & $38,5 \% *$ & \\
\hline Função auditiva expressiva & $33,4 \% *$ & \\
\hline Função visual & $100 \% *$ & \\
\hline \multicolumn{3}{|l|}{ Escala de Gesell e Amatruda } \\
\hline Comportamento comunicativo & 18 meses & \\
\hline Comportamento motor grosseiro & 12 meses & \\
\hline Comportamento motor delicado & 10 meses & \\
\hline Comportamento de linguagem & 6 meses & \\
\hline Comportamento pessoal-social & 14 meses & \\
\hline Categoria de audição & $0^{1}$ & \\
\hline Categoria de linguagem expressiva & $1^{2}$ & \\
\hline Modo de comunicação predominante & $2^{3}$ & \\
\hline
\end{tabular}

* Análise quantitativa dos itens apresentados pela criança em cada função

Legenda: $\mathrm{OD}=$ orelha direita; $\mathrm{OE}=$ orelha esquerda; $\mathrm{dB}=$ decibel; $\mathrm{ARV}=$ audiometria de reforço visual; LDV = limiar de detecção verbal; $\mathrm{AASI}=$ aparelho de amplificação sonora individual; EOE = emissões otoacústicas evocadas; PEATE = potencial evocado auditivo de tronco encefálico; RAEE = resposta auditiva de estado estável; ${ }^{1}$ = não detecta a fala; ${ }^{2}=$ ausência de fala ou vocalizações indiferenciadas; ${ }^{3}=$ comunicação por meio de gestos 
Tabela 2. Resultados da avaliação pré-cirúrgica utilizado para indicação do implante coclear em crianças com paralisia cerebral: aspectos psicológicos, médicos e avaliações por imagem

\begin{tabular}{ll}
\hline Avaliações & Resultados \\
\hline Avaliação psicológica & \\
Entrevista psicológica & Ausência de psicopatologias na criança e na família \\
Grau de permeabilidade da família & $96,4 \%$ \\
Estilo cognitivo da criança & $100 \%$ \\
\hline Avaliações clínicas médicas & Ausência de comprometimentos associados a PC \\
Neurologia & Quadriparesia atetóide de grau moderado, maior prejuízo motor no lado esquerdo \\
& Ausência de contra-indicações à cirurgia de IC \\
Otorrinolaringologia & Espectro da neuropatia auditiva de grau profundo bilateral \\
\hline Avaliações por imagem & Aumento do espaço subaracnóideo frontal bilateral e ausência de lesões isquêmicas \\
RM de crânio & Ausência de anormalidades \\
TC das mastóides & Ausência de anormalidades \\
RM da fossa posterior e regiões temporais &
\end{tabular}

Legenda: $\mathrm{PC}=$ paralisia cerebral; $\mathrm{IC}$ = implante coclear; $\mathrm{RM}$ = ressonância magnética; $\mathrm{TC}$ = tomografia computadorizada

cirurgia e o acompanhamento está sendo realizado sistematicamente no hospital.

\section{DISCUSSÃO}

A avaliação médica e fonoaudiológica para a definição do tipo e grau da perda auditiva nesta criança se diferenciaram das existentes no protocolo padrão, pela particularidade da criança avaliada possuir paralisia cerebral. A bateria de testes e procedimentos foi fundamental para descartar perda auditiva de caráter central. A perda auditiva de etiologia central é considerada contra-indicação ao IC. Em casos de lesões encefálicas extensas, outras regiões corticais, além dos centros motores, podem ser atingidas. Desta maneira, os exames por imagem (RM/TC) foram considerados auxílios valiosos e essenciais para o diagnóstico audiológico.

A aplicação das escalas de desenvolvimento de Gesel e Amatruda ${ }^{(13)}$ e a Escala ELM ${ }^{(14)}$ nos indicou o estado de desenvolvimento do paciente em outros domínios, além das habilidades auditiva e linguística. Neste caso, era esperado encontrar atraso no desenvolvimento global, compatíveis com o quadro de paralisia cerebral, e pontuações rebaixadas nos questionários aplicados, o que de fato foi observado (Tabela 1). Apenas no domínio da função visual o paciente não apresentou atraso em relação à idade cronológica. No questionário PRISE ${ }^{(11)}$, que se refere às vocalizações e habilidades comunicativas, o paciente apresentou pontuação acima de 50\%, reafirmando seu potencial comunicativo.

É importante realçar que um grupo de crianças com PC e deficiência auditiva sempre será considerado um grupo heterogêneo. A única semelhança reside no fato de apresentarem deficiência auditiva, e serem candidatas ao IC. Em contrapartida, as demais características do quadro motor e cognitivo, são distintas, podendo se apresentar potencialmente como variáveis no processo de avaliação e, consequentemente, de habilitação e reabilitação com o IC. Por este motivo, este estudo reforça a necessidade de avaliar integralmente uma criança com PC, em equipe interdisciplinar, considerando-se os aspectos individuais de desenvolvimento de cada criança. Neste estudo, o participante apresentou características favoráveis à indicação do IC.

A maioria das pesquisas internacionais englobaram na amostra de estudo crianças com diferentes deficiências associadas, incluindo a PC como sendo uma delas ${ }^{(2,3,5,7,7)}$. Apenas um artigo tratou exclusivamente do implante coclear em crianças com paralisia cerebral ${ }^{(2)}$. Este estudo avaliou a percepção e a inteligibilidade de fala destas crianças após determinados períodos de uso do IC. No entanto, a respeito do processo de avaliação pré-cirúrgica, não existem informações.

Os artigos sobre implante coclear nesta população de crianças, com PC ou outras deficiências associadas à surdez, possuem como tema central a análise dos benefícios observados após o uso do IC, em relação à percepção e à produção de fala. O processo de avaliação anterior à indicação do IC não é estudado de maneira aprofundada. Desta maneira, é possível deduzir que as avaliações e critérios utilizados para a seleção de crianças com múltiplas deficiências candidatas ao implante coclear, não se diferiram grandemente dos protocolos e critérios já utilizados para crianças com deficiência auditiva isolada, nos diferentes centros onde os estudos foram realizados.

Os critérios de indicação do IC para crianças com PC, neste estudo, não se distinguiram dos já utilizados para crianças com deficiência auditiva isolada ${ }^{(9)}$. Entretanto, o processo de avaliação pré-cirúrgica deve apresentar instrumentos direcionados aos aspectos globais do desenvolvimento, e específicos para cada deficiência associada à surdez, neste caso a PC. Isto será fundamental para estabelecer o prognóstico, bem como para conduzir o processo de orientação e aconselhamento familiar, especialmente no que se refere às expectativas da família.

Para crianças que apresentam paralisia cerebral ou outras deficiências associadas à surdez, a documentação pré-cirúrgica sobre o desempenho funcional em diferentes áreas do desenvolvimento é valiosa e necessária. Pode facilitar a obtenção de medidas para quantificar e qualificar os progressos após o 
uso do IC, e provavelmente gerar discussões muito além do benefício auditivo e de fala para crianças com PC, em especial, quanto à qualidade de vida.

\section{COMENTÁRIOS FINAIS}

Neste estudo de caso, a PC não foi considerada contra-indicação para o IC. O caso descrito apresentou todos os critérios necessários para receber o IC. Entretanto, ressalta-se a importância de uma equipe interdisciplinar para a caracterização do estado de saúde geral, bem como dos níveis de desenvolvimento globais da criança candidata ao IC que apresente em associação a paralisia cerebral ou qualquer outra desabilidade adicional.
Avaliações direcionadas e amplas durante o processo de indicação do IC para este grupo de crianças auxiliam na decisão de indicação ou não indicação do IC, além de trazerem informações úteis para o planejamento terapêutico, e fundamentalmente para enriquecer as orientações dadas aos pais sobre o prognóstico da criança.

\section{AGRADECIMENTOS}

Agradecemos ao Conselho Nacional de Desenvolvimento Científico e Tecnológico - CNPq, pela bolsa de Iniciação Científica concedida para realização desta pesquisa, sob processo número 116125/2008 0 .

\begin{abstract}
Cerebral palsy (CP) is the most common motor disorder in childhood, and is usually associated with other disabilities, including hearing impairment. Studies regarding the indication of cochlear implant (CI) for children with cerebral palsy and hearing impairment are restricted internationally, and, in Brazil, non-existent. Therefore, the purpose of this study was to describe the process of pre-surgical assessment of a child with $\mathrm{CP}$ who was a candidate for cochlear implantation. The study was conducted with a boy, 2 years and 6 months old, with $\mathrm{CP}$ and bilateral hearing impairment. The pre-surgical protocol followed the procedures already used at the Hospital for Rehabilitation of Craniofacial Anomalies, including: speech-language pathology and audiological, neurological, otorhinolaryngological, and psychological evaluations through clinical observations, questionnaires, and imaging findings. The patient was diagnosed with profound and bilateral auditory neuropathy spectrum disorder, moderate quadriparesis athetoid, global developmental delay (except in visual function), presence of intentional communicative behavior, good social contact, absence of intellectual impairments or other additional disabilities within the clinical framework of CP, and a family integrated to the treatment. Based on the results obtained, the CI was recommended to this patient, who is currently enrolled in a systematic monitoring program. This study highlights the importance of the pre-surgical protocol for children with $\mathrm{CP}$ who are candidates for CI. Through assessment tools directed to the global aspects of development, one can obtain specific information that improve parent counseling regarding the child's prognosis and make it possible to trace actual hearing rehabilitation goals.
\end{abstract}

Keywords: Cochlear implantation; Cerebral palsy; Hearing loss; Child; Evaluation; Developmental disabilities

\title{
REFERÊNCIAS
}

1. Lamônica DA. Estimulação da linguagem em crianças com paralisia cerebral. São Paulo: Pulso; 2008. Estimulação da linguagem: aspectos teóricos e práticos; p. 163-77.

2. Bacciu A, Pasanisi E, Vincenti V, Ormitti F, Di Lella F, Guida M, et al. Cochlear implantation in children with cerebral palsy. A preliminary report. Int J Pediatr Otorhinolaryngol. 2009;73(5):717-21.

3. Berrettini S, Forli F, Genovese E, Santarelli R, Arslan E, Chilosi AM, et al. Cochlear implantation in deaf children with associated disabilities: challenges and outcomes. Int J Audiol. 2008;47(4):199-208.

4. Moret AL, Bevilacqua MC, Costa OC. Implante coclear: audição e linguagem em crianças deficientes auditivas pré-linguais. Pro-Fono. 2007;19(3):295-304.

5. Wiley S, Jahnke M, Meinzen-Derr J, Choo D. Perceived qualitative benefits of cochlear implants in children with multi-handicaps. Int $\mathbf{J}$ Pediatr Otorhinolaryngol. 2005;69(6):791-8.

6. Nascimento LT. Uma proposta de avaliação da linguagem oral [monografia]. Bauru: Universidade de São Paulo, Hospital de Reabilitação de Anomalias Craniofaciais; 1997.

7. Daneshi A, Hassanzadeh S. Cochlear implantation in prelingually deaf persons with additional disability. J Laryngol Otol. 2007;121(7):635-8.
8. Waltzman SB, Scalchunes V, Cohen NL. Performance of multiply handicapped children using cochlear implants. Am J Otol. 2000;21(3):329-35.

9. Bevilacqua MC, Moret AL. Reabilitação e implante coclear. In: Lopes Filho O. Tratado de fonoaudiologia. Ribeirão Preto: Tecmedd; 2005. p. 423-36.

10. Castiquini EA, Bevilcqua MC. Escala de integração auditiva significativa: procedimento adaptado para a avaliação da percepção da fala. Rev Soc Bras Fonoaudiol. 2000;4(6):51-60.

11. Kishon-Rabin L, Taitelbaum-Swead R, Ezrati-Vinacour R, Hildesheimer M. Prelexical vocalization in normal hearing and hearing-impaired infants before and after cochlear implantation and its relation to early auditory skills. Ear Hear. 2005;26(4 Suppl):17S-29S.

12. Padovani CM, Teixeira ER. Using the MacArthur Comunicative Development Inventories (CDI'S) to assess the lexical development of cochlear implanted children. Pro-Fono. 2004;16(2):217-24.

13. Gesell A. Gesell e Amatruda: diagnóstico do desenvolvimento: avaliação e tratamento do desenvolvimento neuropsicológico do lactente e da criança pequena, o normal e o patológico. 3a ed. São Paulo: Atheneu; 2000.

14. Coplan J. Early Language Milestone Scale. Austin: Pro-Ed; 1993. 